\title{
A Framework for Generating Diverse Haskell-I/O Exercise Tasks
}

\author{
Oliver Westphal \\ University of Duisburg-Essen \\ oliver.westphal@uni-due.de
}

\begin{abstract}
We present the design of a framework to automatically generate a large range of different exercise tasks on Haskell-I/O programming. Automatic task generation is useful in many different ways. Manual task creating is a time consuming process, so automating it saves valuable time for the educator. Together with an automated assessment system automatic task generation allows students to practice with as many exercise tasks as needed. Additionally, each student can be given a slightly different version of a task, reducing issues regarding plagiarism that arise naturally in an e-learning environment. Our task generation is centered around a specification language for I/O behavior that we developed in an earlier work. The task generation framework, an EDSL in Haskell, provides powerful primitives for the creation of various artifacts, including program code, from specifications. We will not go into detail on the technical realization of these primitives. This article instead showcases how such artifacts and the framework as a whole can be used to build exercise tasks templates that can then be (randomly) instantiated.
\end{abstract}

\section{Introduction}

We have recently designed and implemented a language for the specification of console I/O programs [9][10] that allows the formulation of desired I/O behavior and probabilistic testing of actual programs, written in Haskell, against the specified behavior. We built this language to bring our testing capabilities of exercises on Haskell I/O more in line with how one can test exercises on pure programs using, for example, QuickCheck [1]. The testing capabilities gained this way are used in the e-learning system $[4,7]$ accompanying our course on programming paradigms.

Instead of only automatically checking exercise submissions against desired behavior, we also want to automatically generate the tasks themselves. Automatic task generation, among other things, gives students more material to practice with, without the need for an instructor to write all of this material by hand. Because our specification language is designed to describe the I/O behavior of programs, we will (only) end up with automatic task generation for tasks on Haskell I/O in this work.

Hand-written tasks are usually very heavy in terms of verbal descriptions of what a student has to do in order to solve the task. This is a major problem when 
trying to generate tasks automatically, since natural language generation is not exactly easy. Because of this, many task generation systems rely on templates that define a fixed (verbal) framing of a task containing parameters that can be filled in in order to get the finial task. Choosing different parameters to fill into these holes, either randomly or by drawing from a fixed set of possibilities, then results in different tasks. Whether such a template, and a fixed verbal framing, is easy to write depends on the domain for which tasks should be generated. For example, it is easy to do so for many different math tasks ("Solve for $x$." etc.), but for programming tasks finding a fixed and general verbal framing is not as easy. Essentially we need a way to describe varying requirements in a precise but non verbal way. To achieve such a description our approach uses different artifacts, like actual program code, derived from the requirements underlying the task. As an example, take the following tasks:

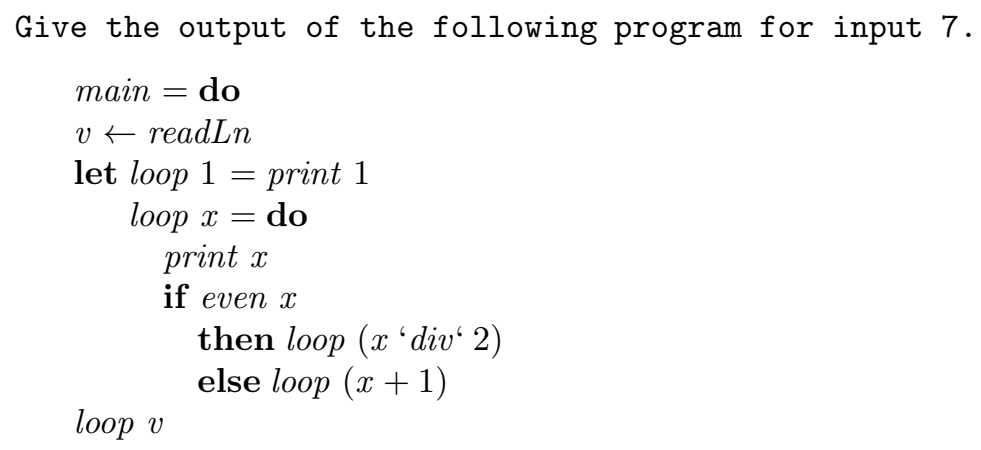

Here the verbal description will not change, no matter what we give as the program text. As we will see in section 4, there is a wide variety of possibilities to express tasks in this artifact driven style: from simple decision tasks over program comprehension to full blown programming exercises.

We present a newly developed EDSL, in Haskell, for describing such task templates. Our approach is built on top of the existing implementation of the specification language from our previous work. We start from a specification, hand-written or generated randomly, and use the EDSL to derive different artifacts, like example runs and sample solutions, that we can use for building task templates. Now a task basically consists of giving students one of these artifacts and ask for the other. Additionally we also have the testing capabilities of our implementation of the specification language at our disposal. Therefore we can also automatically check solution candidates for the generated tasks for correctness.

We will not go into the technical details of how we create these artifacts in the implementation. Instead this presentation focuses on the framework's versatility in expressing interesting task ideas and generating variations.

We will start by looking at how to encode task templates with Haskell types in a domain independent way. Next we give a short overview of the previously introduced specification language. Using these two components together, we show how to design a diverse range of exercise tasks on Haskell-I/O. 


\section{Describing parameterized tasks}

First we need to define how we encode a task template. In its simplest form it is a function from some concrete values to an instance of the task. That is, the argument of the function stands for the parameterized parts of the template.

We represent a task instance as a pair of a textual description and a requirement to what constitutes a correct solution.

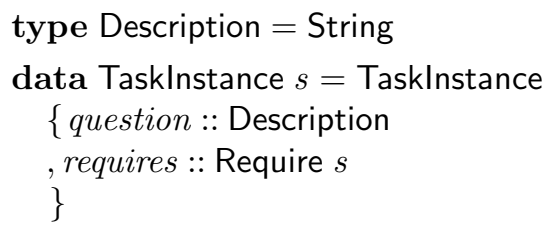

A Tasklnstance is polymorphic in the type of solutions it expects. Require $s$ then takes a value of type $s$ and decides whether that value is a correct solution. Instead of simply returning a value of type Bool we use QuickCheck's Property type for reasons that will become clear later.

$$
\text { newtype Require } s=\text { Require }\{\text { check }:: s \rightarrow \text { Property }\}
$$

Now we can define a parameterized task as a simple function. The basic setup for tasks is not yet specific to Haskell I/O, so we as an example define a very simple task for adding two numbers:

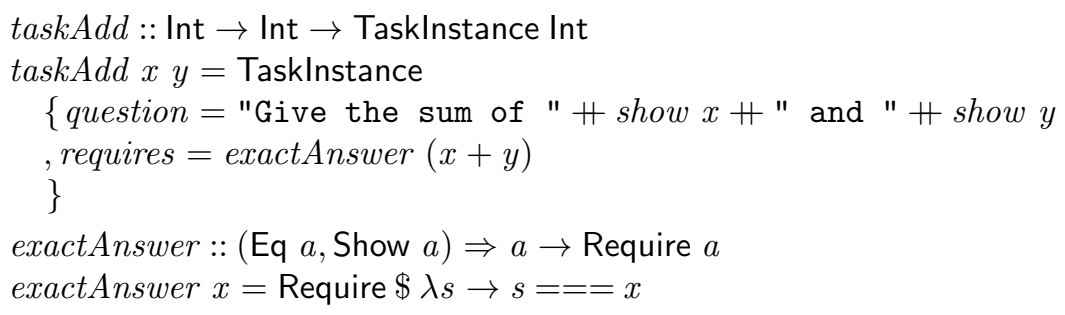

We define the requirement for an exact answer in terms of QuickCheck's $(===)$ operator that produces an informative feedback message in case the test fails. For example, giving the wrong solution to an instance of the above tasks might result in the following error when testing the requirement:

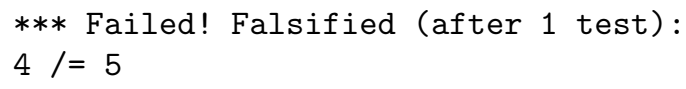

We can therefore leverage QuickCheck to provide students with feedback on their solution.

Note that since we use actual Haskell functions to describe parameterized tasks we can in principle have different instances based on what parameters we choose to fill the holes in the task description with. We can take this idea one step further and not only choose Tasklnstance's based on the parameters, but 
add random variations to the instances themselves. With this extension we get the following datatype for templates:

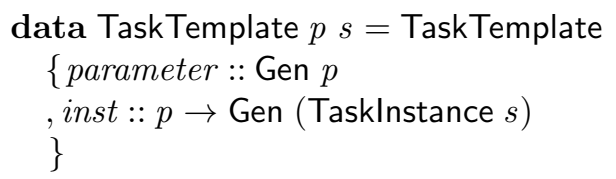

Here Gen is QuickCheck's generator monad type, representing the randomized variations in the parameters and task instance. Every template for a task consists of a generator for parameters and a parameterized generator for an actual instance. To instantiate a template we first generate a parameter value and then the instance body:

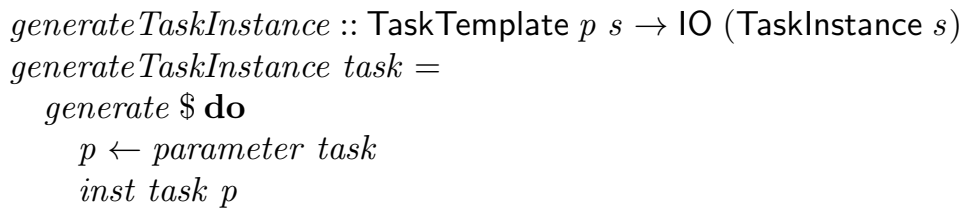

Additionally, given a way to retrieve a solution candidate from somewhere, we can generate a TaskInstance and check the solution candidate against the requirements like this:

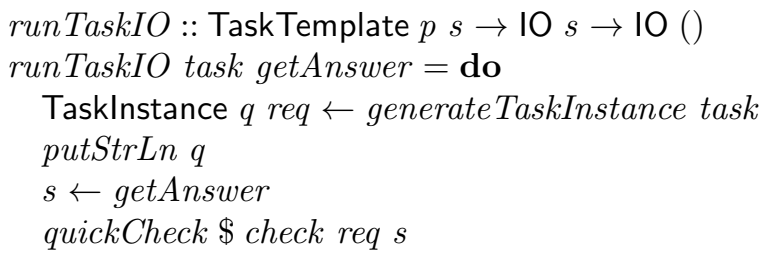

For convenience we define some combinators for defining templates and instances that make these definitions more directly readable.

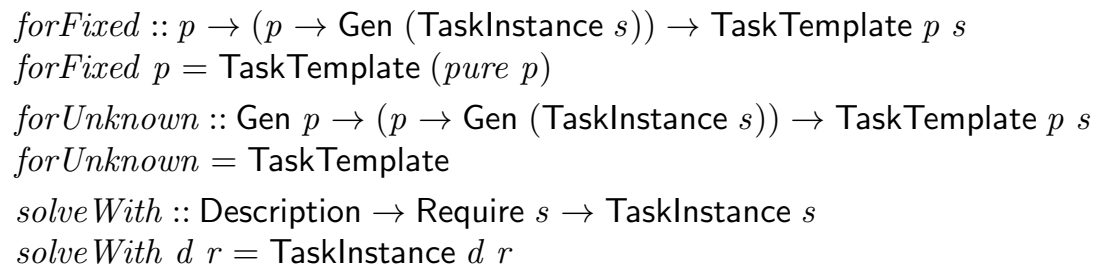

\section{The specification language}

Before diving into the description of I/O tasks, we give a short summary of the specification language, introduced in our previous work [9], as it forms the basis of the presented approach. Specification expressed in the language describe 
program behavior in terms of the traces, i.e, sequences of read and written values, a program should produce. By checking for different (carefully randomized) inputs wether a program produces traces satisfying the specification, we can automatically test programs against specifications. The specifications themselves let us state task requirements in a precise way and the ability to automatically test programs against a specified behavior enables automatic grading of exercise tasks.

We will not present the language in the formal way of [9], but instead use the Haskell embedding, since this embedding is what we are going to use. Specifications are built from three atomic specifications, a branching and a loop construct and sequential composition. The atomic specifications are:

- The empty specification, representing a program with no I/O behavior.

$$
\text { nop :: Specification }
$$

- Reading in a value drawn from a set of possible values.

$$
\text { readInput }:: \text { Varname } \rightarrow \text { ValueSet } \rightarrow \text { Specification }
$$

Here ValueSet is a type describing which values we expect to be read in with the respective input action. We will use the predefined values ints ::ValueSet and nats :: ValueSet for integers and natural numbers.

It is important to note that variables, in the specification language, always store all values previously read into them. That is, each variable is associated with a historic list of values. There are two ways to access a variable in a term:

$$
\begin{aligned}
& \text { getAll }:: \text { Varname } \rightarrow \text { Term }[a] \\
& \text { getCurrent }:: \text { Varname } \rightarrow \text { Term } a
\end{aligned}
$$

We either access the complete list of all read values into a variable or we access only the current or most recent value, i.e., the last element of the stored list.

- Printing the result of evaluating some term on the current variable environment, potentially decorated with some extra textual pattern

$$
\text { writeOutput :: [OutputPattern] } \rightarrow[\text { Term } a] \rightarrow \text { Specification }
$$

The list parameters here stand for different output possibilities, thereby introducing a form of nondeterminism. OutputPattern describes what kind of output we expect from a program. These patterns can reference the values of Terms in the second parameter of writeOutput. We will not go into detail on these types. Their usage in the examples should be intuitive. However, we rely on being able to not only evaluate terms but also inspect the syntactic structure of terms. Therefore, the implementation provides functions to convert a Term into an abstract syntax tree. Essentially, a value of type Term $a$ is a pair of a value of type $a$ and it's syntactic representation. 
Sequential composition of Specifications forms a Monoid, which gives us $(<>):$ : Specification $\rightarrow$ Specification $\rightarrow$ Specification for combining smaller Specifications into larger ones.

In addition to the atomic specifications we have constructs for conditions and looping. To allow for conditional behavior, we use:

$$
\text { branch }:: \text { Term Bool } \rightarrow \text { Specification } \rightarrow \text { Specification } \rightarrow \text { Specification }
$$

Depending on the Boolean value the condition evaluates to, either the left or right specification is required to hold. Here the left branch corresponds to False and the right to True, due to the way the operator is notated in the original definition.

To repeat a certain (sub)specification, we use:

$$
\begin{aligned}
& \text { tillExit :: Specification } \rightarrow \text { Specification } \\
& \text { exit :: Specification }
\end{aligned}
$$

The specification tillExit $s$, for some $s$ :: Specification, results in repeatedly requiring the behavior specified by $s$ until somewhere in $s$ we hit an occurrence of the loop end marker exit, which leads to immediate termination of the loop. Note that there can be more than one such marker inside $s$.

As an example, the following specification describes the behavior of reading in a natural number followed by that many additional integers and then printing the sum of these integers:

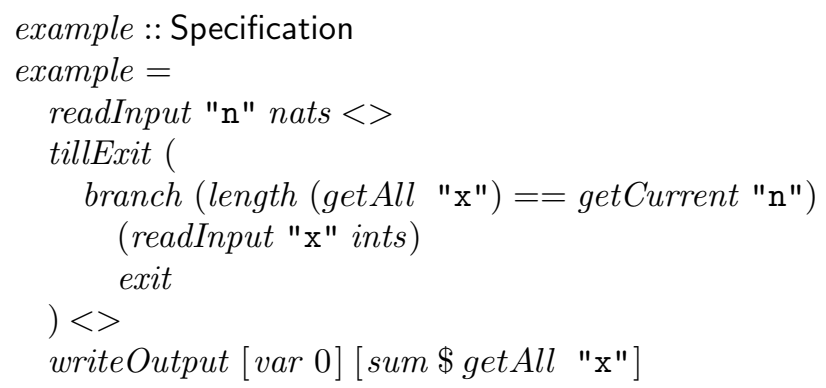

Note that length, sum and $==$ are not the functions of the same names from the Haskell prelude. Instead they are syntactically inspectable versions, over values of type Term, with equivalent behavior. This inspection is needed to built printable programs from specifications for the use in tasks descriptions.

\section{Tasks on Haskell I/O}

The testing approach of the specification framework relies on programs being represented in an inspectable version of the standard Haskell IO monad $[9,6]$.

$$
\text { type Program }=I O_{r e p}()
$$


Together with a Specification we can construct a Property that checks whether the program satisfies the behavior defined by the specification.

$$
\text { fulfills :: Program } \rightarrow \text { Specification } \rightarrow \text { Property }
$$

Using fulfills, which is provided by the implementation of [9], we can construct our first I/O specific requirement for a correct task solution.

$$
\begin{aligned}
& \text { behavior }:: \text { Specification } \rightarrow \text { Require Program } \\
& \text { behavior } s=\text { Require ('fulfills's) }
\end{aligned}
$$

We can also build a requirement to check a given interaction trace, i.e. the sequence of values read and written by the program, against some specification, using an implementation of the accept function from [9].

$$
\begin{aligned}
& \text { sampleTrace }:: \text { Specification } \rightarrow \text { Require Trace } \\
& \text { sampleTrace } s=\text { Require } \$ \lambda t \rightarrow \text { property } \$ \text { accept } s t
\end{aligned}
$$

We will define additional requirements as we discuss the various example tasks.

In addition to requirements we also need something to build descriptions from. Our system offers essentially two options to produce such artifacts from specifications: program code and example traces. In both cases the system provides parameterized generators to build the artifacts.

$$
\begin{aligned}
& \text { haskellProgram }:: \text { Specification } \rightarrow \text { Gen Description } \\
& \text { pythonProgram }:: \text { Specification } \rightarrow \text { Gen Description } \\
& \text { exampleTraces }:: \text { Specification } \rightarrow \text { Int } \rightarrow \text { Gen [Trace] }
\end{aligned}
$$

These generators will generate programs that fulfill the given specification or a number of example traces that a program with the specified behavior could produce. For program code we mainly use Haskell code in our tasks, but it is also useful to have access to code in other languages and paradigms. For example, we sometimes use Python code, since that is the language our students know from a previous course, to highlight how I/O functions differently in Haskell compared to an imperative language.

We will not discuss the technical details of the code generation, as our focus is on showcasing the different types of tasks that are expressible in the system. There is, however, one aspect of this generation that is important for our presentation: Even though we are going to treat the generation of code as a primitive operation provided by the framework, in the actual implementation the generation is adjustable in different ways. Most importantly we can generate code in different programming languages, by defining rendering functions from an internal representation of programs to the desired target language. With this mechanism we can also generate partial programs by omitting certain parts of the internal representation, either deterministically or randomized. Such partial programs are useful in a lot of different ways, as we will see later.

With all of these tools we can now define a diverse range of task templates. We will mainly use specifications, i.e. behavior, as parameters for our tasks and 
therefore assume the existence of some suitable generators. A basic sketch of how to write such a generator can be found in [10]. For our task templates we assume the existente of these two generators.

$$
\begin{aligned}
& \text { randomSpecification :: Gen Specification } \\
& \text { similarSpecifications :: Gen (Specification, Specification) }
\end{aligned}
$$

The first generator simply generates a random, but meaningful, specification. The second generator generates a pair of specifications that are closely related but have different behavior.

The source code of the implementation and all examples from this paper, can be found at https://github.com/fmidue/IOTasks. ${ }^{1}$

\subsection{Simple program comprehension tasks}

To get started exploring the task design space for, we can build a task around the simple question "What does this program do?". An instance of such a task would be:

Give the interaction trace of the following program for input(s)

1,9 .

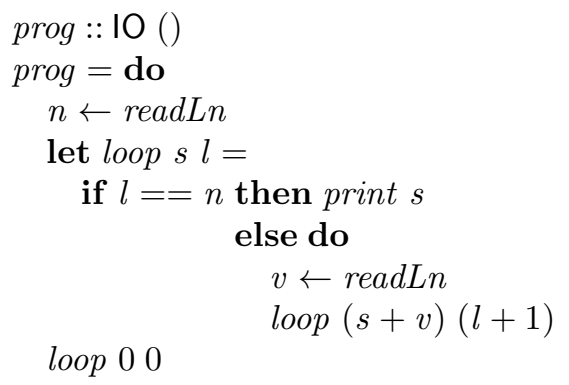

The template expression for such tasks looks like this:

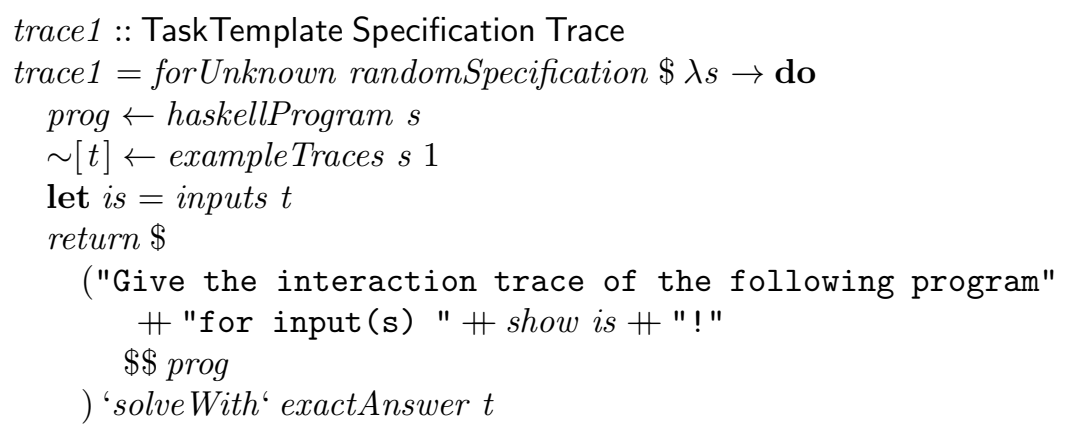

${ }^{1}$ The repository also contains instructions explaining how to, for the given examples, generate and inspect task instances that have been omitted from this presentation. 


$$
\begin{aligned}
& (\$ \$):: \text { Description } \rightarrow \text { Description } \rightarrow \text { Description } \\
& s \$ \$ t=s+\text { "\n" }+t
\end{aligned}
$$

An even more basic variation of this task would be to not give an input sequence at all and simply ask for any possible interaction trace the program can produce. For a more involved task we can generate two similar looking programs with different behavior and let students find an input sequence for which the given programs exhibit different I/O behavior.

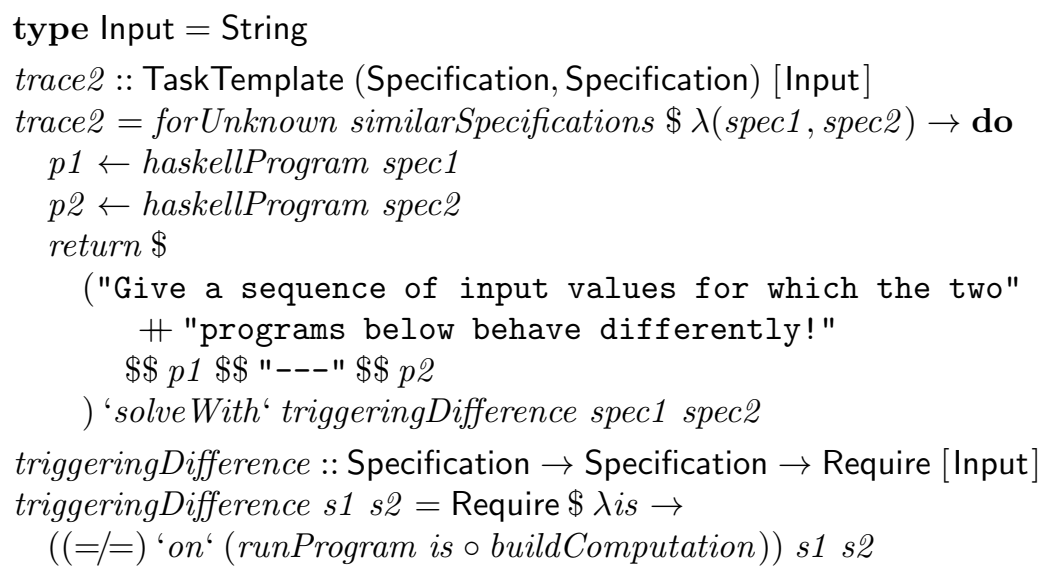

In order to check the requirement for this tasks, we here make use of the ability of the specification framework to derive an executable program from a specification via the buildComputation function. Details on this can be found in [10].

\subsection{Program writing tasks}

To explore other kinds of task, we can reverse the question underlying the example above. The reverse direction of "What does this program do?" is "Do this with a program!", where "this" would refer to a given description of behavior. Producing such descriptions in a precise and automatic way is exactly what the artifact primitives are for. These primitives are the central ingredients that enable us to describe the diverse set of tasks we will see in this section.

A direct reversal of "What is the behavior of this program for input X?" is the, arguably, not very interesting task "Write a program that does Y for input X!". But generalizing from a single input to a larger set of "program runs", i.e. traces, we immediately get a more compelling template:

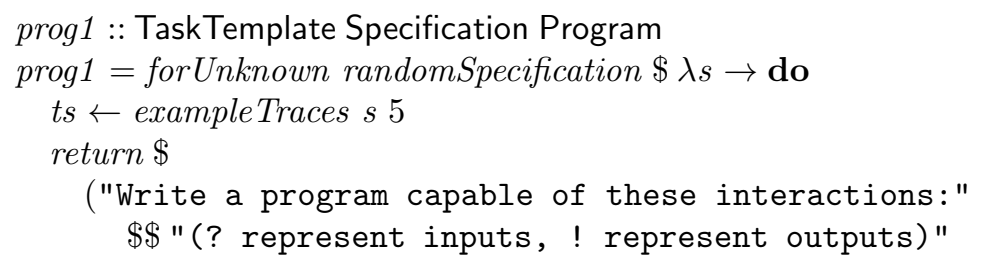




$$
\begin{aligned}
& \text { \$\$ unlines ( map show ts) } \\
& \text { )'solveWith' producingTraces ts } \\
& \text { producingTraces }::[\text { Trace }] \rightarrow \text { Require Program } \\
& \text { producingTraces } t s=\text { Require } \$ \lambda p \rightarrow \\
& \text { property } \$ \text { all }(\lambda t \rightarrow \text { runProgram }(\text { inputs } t) p==t) \text { ts }
\end{aligned}
$$

The requirement here checks that the solution produces exactly the given traces for the respective inputs. An instance of this task could look like this:

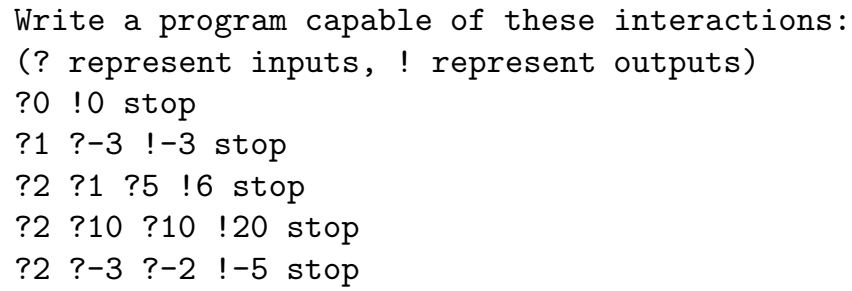

With such a task, however, we can no longer expect a solution to have the exact same behavior as our underlying specification. The description essentially gives a list of unit tests a correct solution has to fulfill. The fact that there is an underlying specification guarantees that abstraction is possible, i.e, there is at least on program that solves the task without simply hard-coding the given interactions. Simply writing such a program that only works on the given inputs and crashes on all others, however, would still be a correct solution with regard to the task requirement. If we do not want students to write such solutions, we can either give a very large number of program runs, making it unpractical to explicitly implement each case, or we add some restrictions ruling out such solutions. One possibility is to provide parts of the solution as a skeleton to complete. This can either be a general skeleton with a specific style, or an actual part of a solution.

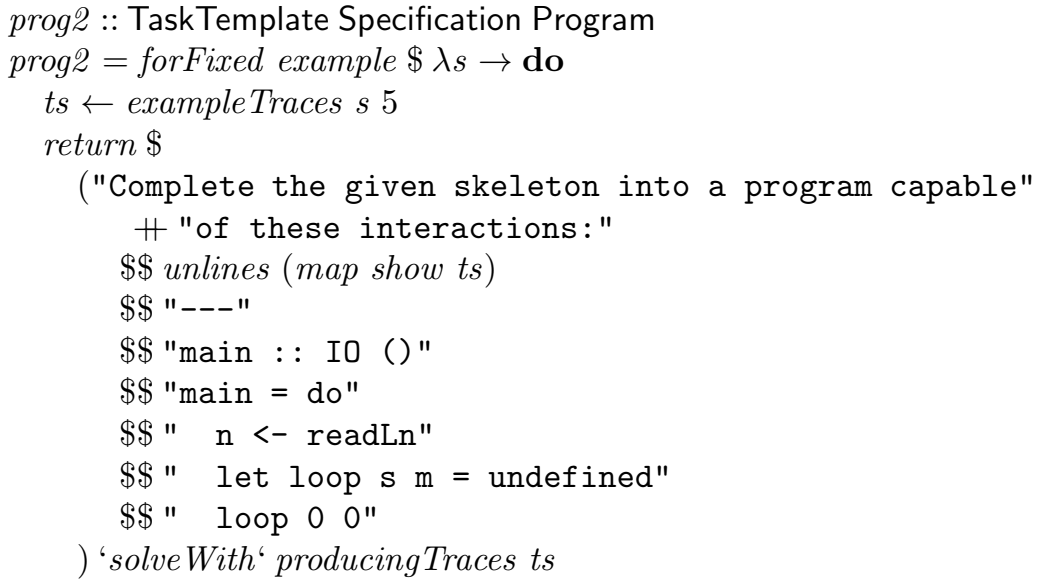

Note that adherence to the skeleton is unchecked here. In a general setting we would add an additional requirement to the template, but we currently already 
pose hand-written versions of such tasks and our current automatic grading setup for Haskell tasks already supports such checks [4,7]. For example, we already give students programs such as the one above (together with a verbal task description), where they have to replace all occurrences of undefined by something appropriate. The system then makes sure that only occurrences of undefined are replaced.

Additionally, we here use a fixed specification, example from page 6, instead of a random one. Using a random specification, without any further restrictions, potentially results in an unsolvable task since the skeleton can have the wrong structure for the underlying behavior.

Instead of using a fixed skeleton to guide a solution into a certain direction, we can also create the skeleton from a generated program. As described above, internally program generation goes through a rendering step in which the final appearance of the code is determined. We can create partial programs during this step, for example, by replacing certain parts of the program with holes. This way we can create a skeleton that can always be complete to a valid solution. Since we do not concern ourselves with the implementation details of this rendering, we simply assume that we have an implementation of the following generator available:

$$
\text { haskellWithHoles :: Specification } \rightarrow \text { Gen Description }
$$

Besides producing program skeletons, such partial programs are useful for other tasks as well. For example, we can test students abilities to produce syntactically or well typed programs by letting them complete a partial program.

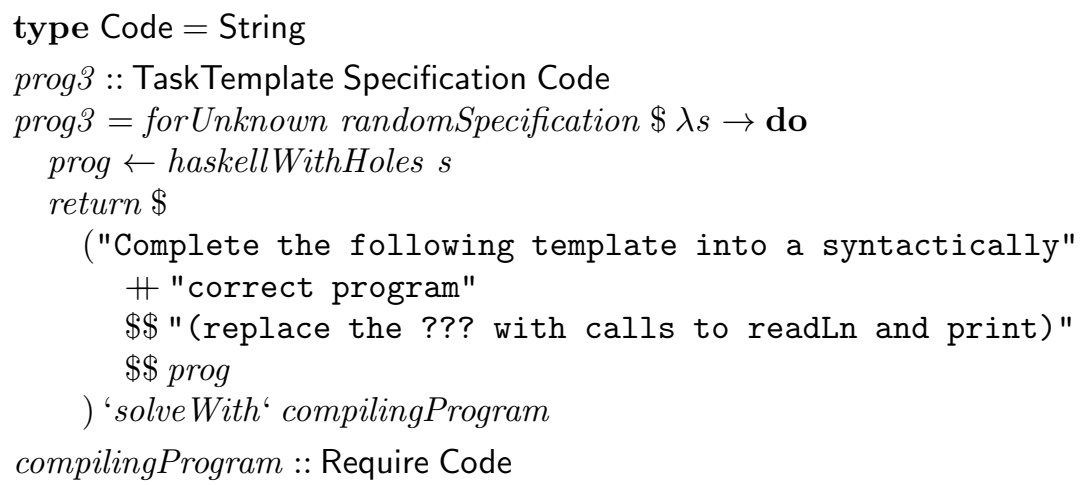

The way the requirement is implemented, depends on the setting in which such a task is used. In the context of our current e-learning setup every submission is already automatically check for compiler errors when submitted. That means, we can implement the requirement with a property that always succeeds, as no noncompiling solution will ever trigger the actual test. Also note that, in contrast to previous tasks, we here specify a requirement on the textual representation of a program instead of the "program-value" itself.

Another area where inspection of the program text is useful are requirements that enforce a solution to use, or not use, certain function or idioms. For example, 
we can pose a task to rewrite some program that uses a list to accumulate values, and then does some computation expressible as a fold on this list, into a version not using any list, but instead directly carrying out the computation without first accumulating values. Of course such a rewrite should preserve the original program's behavior. Therefore, the task has requirements on both the actual program as well as its textual representation.

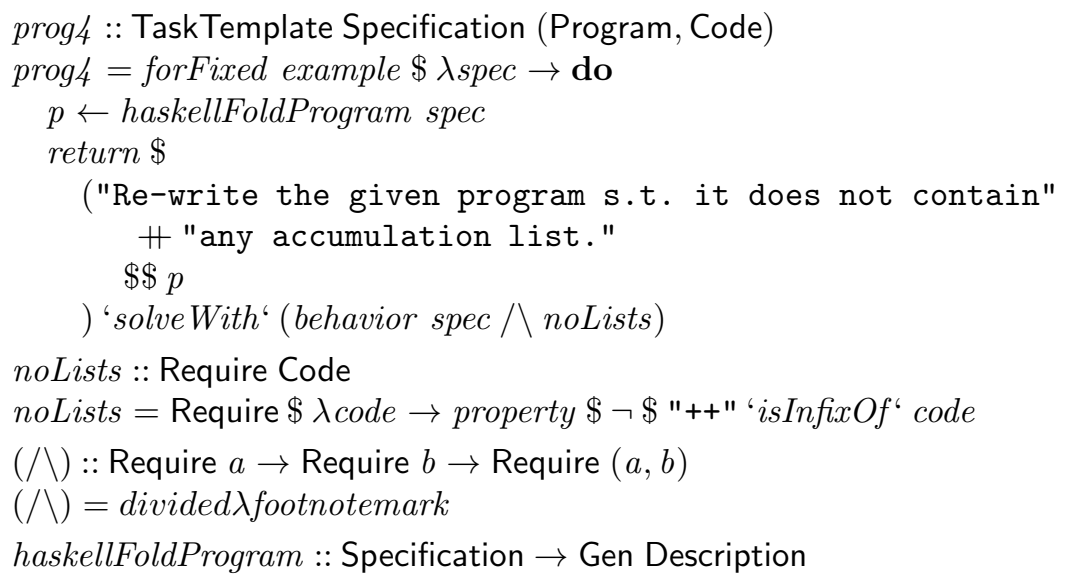

Once again, the details on how haskellFoldProgram is implemented internally are outside the scope of this presentation. Almost none of the above tasks lets students start writing a program on a blank page. Always some part of a solution is already given. To a certain degree this is unavoidable in our approach to task generation, since we need a (non-verbal) description of the task's requirements. But the amount of information about a solution present in this description can be varied. For a free-form style programming task we can, as an example, give the behavior to be implemented in a different programming language, ideally using a different paradigm. In our case, we use Python code for this purpose. Our students learn Python as their first language and are therefore familiar with it. This gives us the opportunity to precisely state what behavior a correct solution has without giving away too much information. Mainly because I/O programs in Haskell usually have a different structure compared to imperative languages (e.g. recursive functions vs. explicit loops).

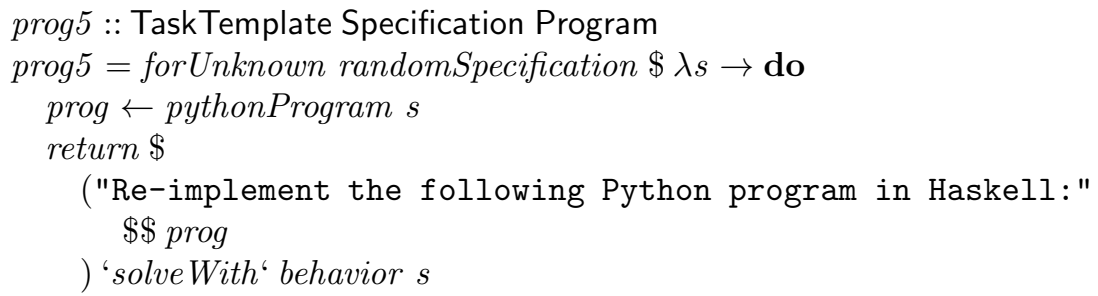

\footnotetext{
${ }^{1}$ Require $s$ is a Divisible, Contravariant functor in $\mathrm{s} .(/)$ therefore simply is divided from Data.Functor.Contravariant.Divisible (see https://hackage.haskell.org/package/ contravariant-1.5.2/docs/Data-Functor-Contravariant-Divisible.html).
} 
From an instance of this task one can gather almost no information on what a Haskell solution could look like:

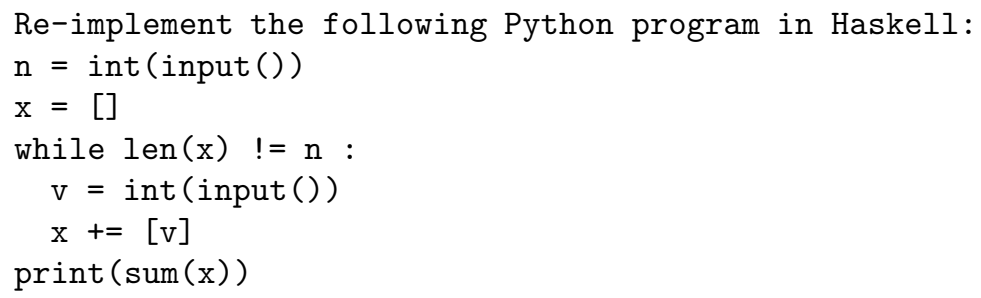

\subsection{Decision tasks}

The examples above always ask students to provide either a program or some trace(s). In most cases the respective other artifact was provided as the requirement description. That is, for a given program a trace needs to be given or vice versa. It might seem that these two options are the only possible ways to pose tasks in the presented framework, but there is a third possibility. Instead of asking for a program or a trace we can instead ask students to make a decision. This decision can be a simple binary choice as well as some multiple-choice questions. The general approach for constructing such tasks is giving students two (or more) artifacts and asking them whether these artifacts originated from the same behavior. To illustrate this type of task let us look at two examples.

First, a simple binary choice would be a task asking whether two given programs have the same behavior.

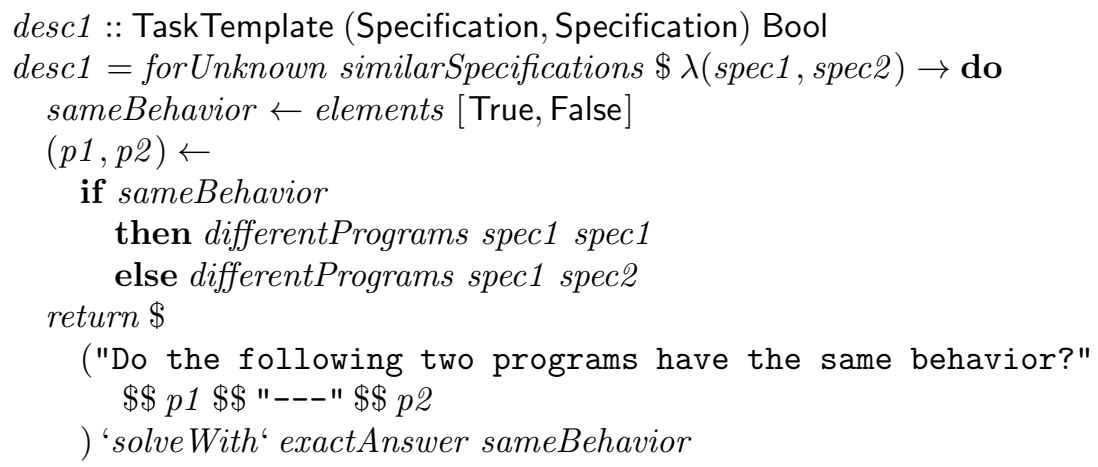

For this template we need to generate two different programs from either the same or two different specifications. ${ }^{2}$ Generating two different programs is straight forward given our primitives and the QuickCheck generator API.

$$
\begin{aligned}
\text { differentPrograms } & :: \text { Specification } \rightarrow \text { Specification } \\
& \rightarrow \text { Gen (Description, Description) }
\end{aligned}
$$

\footnotetext{
${ }^{2}$ Technically we do not need to use differentPrograms to generate two programs with different behavior. Any well behaving implementation of similarSpecification should not generate two specifications that can produce the same program.
} 


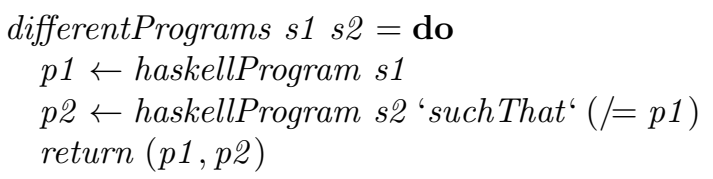

However, in practice one would probably want some additional control over how the two programs actually differ.

As mentioned, we can also build multiple-choice tasks. Here it is again helpful to have randomness available when constructing a task instance. We can define a generator that takes the number of choices we like to provide as well as two lists of correct and incorrect answers. The generator then constructs a selection of choices and also provides the indices of the correct answers.

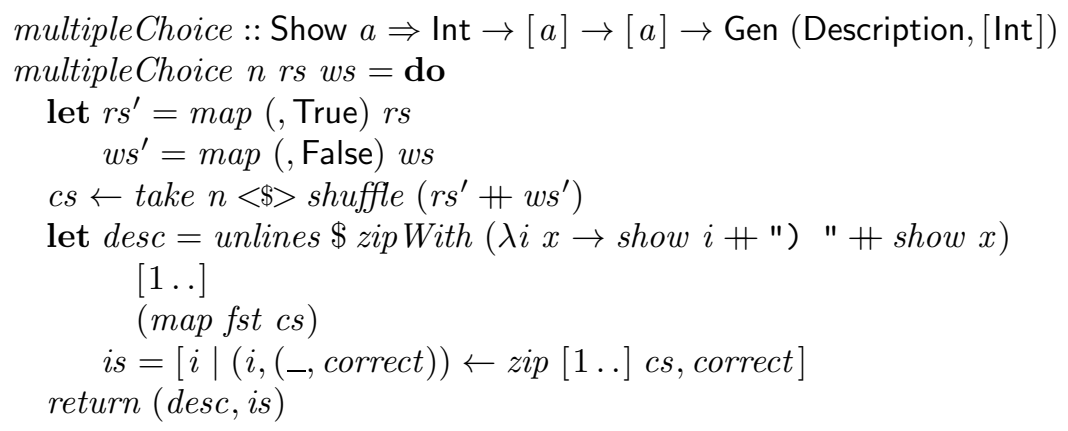

With such a generator we can then easily define a task that, for example, asks to choose which traces a given program can produce.

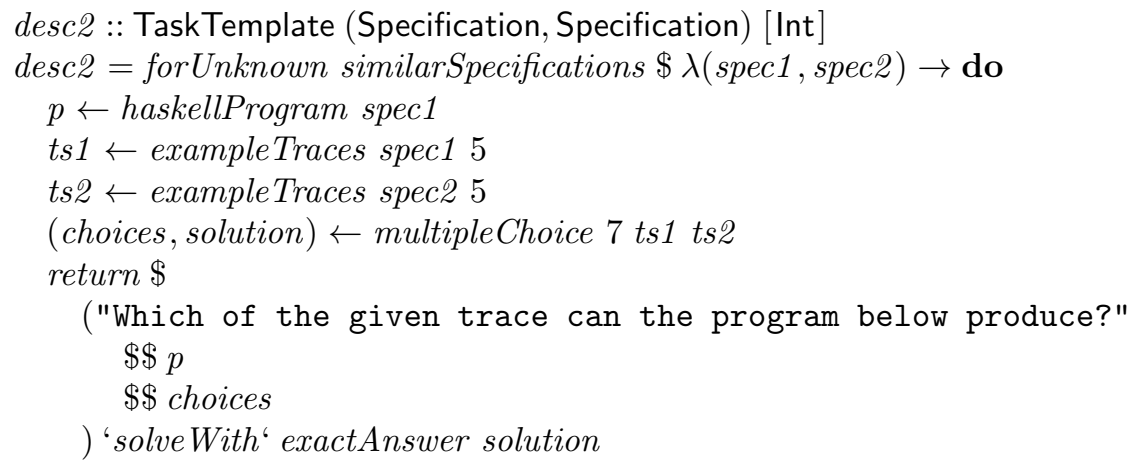

\section{Related Work}

Tools for automatic task generation exist in a variety of different application areas, for example, general science questions [8], math related exercises [2] and programming tasks $[3,5]$. Most approaches use some form of template together with a parameter generator to automatically create new task. Especially for 
natural language tasks, some systems can generate tasks from existing databases of domain specific text [8], but most systems for generating programming tasks rely on templates. In contrast to our flexible EDSL approach most systems use external templates, with varying degrees of expressivness, that are provided as inputs to the task generation. Consequently, they usually come coupled with a fully fleshed out e-learning environment.

\section{Conclusion \& Future work}

We have presented a framework that allows us to describe a diverse range of exercise templates on Haskell I/O. All of the variety stems from the domain specific primitives providing the different artifacts the templates are built around. These artifacts are used as stand-ins for verbal descriptions, allowing us to precisely state task requirements even in the case of randomization of the underlying behavior of a task. Tasks in the presented style differ from traditional hand-written tasks, while still offering a lot of flexibility when designing tasks. The difference to hand-written tasks is also not necessarily a restriction one has to work around, but enables new and interesting task designs. To the best of our knowledge this usage of automatically derived artifacts is a novel approach to automatic task generation.

We have not yet had the opportunity to use the approach in practice. We plan to do a systematic exploration of the design space of exercise task as preparation for the next iteration of our programming paradigms course. Especially, we want to evaluate which tasks help students the most when learning Haskell I/O. We plan to use the results of such a survey to craft automatic assessments for a range of different Haskell-I/O related skills that amplifies students understanding of the subject. Also the question of how to write good generators for the specifications underlying each task is something that should be investigated further, as they have a big influence on the quality of task instances.

\section{References}

1. Claessen, K., Hughes, J.: QuickCheck: a lightweight tool for random testing of Haskell programs. In: International Conference on Functional Programming, Proceedings. pp. 268-279. ACM (2000). https://doi.org/10.1145/351240.351266

2. Kurt-Karaoglu, F., Schwinning, N., Striewe, M., Zurmaar, B., Goedicke, M.: A framework for generic exercises with mathematical content. In: 2015 International Conference on Learning and Teaching in Computing and Engineering, LaTiCE 2015, Taipei, Taiwan, April 9-12, 2015. pp. 70-75. IEEE Computer Society (2015). https://doi.org/10.1109/LaTiCE.2015.11, https://doi.org/10.1109/ LaTiCE. 2015.11

3. Mosbeck, M., Hauer, D., Jantsch, A.: VELS: VHDL e-learning system for automatic generation and evaluation of per-student randomized assignments. In: Nurmi, J., Ellervee, P., Mihhailov, J., Jenihhin, M., Tammemäe, K. (eds.) 2018 IEEE Nordic Circuits and Systems Conference, NORCAS 2018: NORCHIP and 
International Symposium of System-on-Chip (SoC), Tallinn, Estonia, October 3031, 2018. pp. 1-7. IEEE (2018). https://doi.org/10.1109/NORCHIP.2018.8573455, https://doi .org/10.1109/NORCHIP. 2018.8573455

4. Rahn, M., Richter, A., Waldmann, J.: The leipzig autotool e-learning/e-testing system. In: Symposium on Math Tutoring, Tools and Feedback. Open Universiteit Nederland. (2008), http://www.imn.htwk-leipzig.de/ waldmann/talk/ 08/ou08/tool.pdf, accessed July 2020

5. Striewe, M., Balz, M., Goedicke, M.: A flexible and modular software architecture for computer aided assessments and automated marking. In: Cordeiro, J.A.M., Shishkov, B., Verbraeck, A., Helfert, M. (eds.) CSEDU 2009 - Proceedings of the First International Conference on Computer Supported Education, Lisboa, Portugal, March 23-26, 2009 - Volume 2. pp. 54-61. INSTICC Press (2009)

6. Swierstra, W., Altenkirch, T.: Beauty in the beast. A Functional Semantics for the Awkward Squad. In: Haskell Workshop, Proceedings. pp. 25-36. ACM (2007). https://doi.org/10.1145/1291201.1291206

7. Waldmann, J.: Automatische erzeugung und bewertung von aufgaben zu algorithmen und datenstrukturen. In: Proc. ABP, CEUR WS vol. 2015 (2017)

8. Welbl, J., Liu, N.F., Gardner, M.: Crowdsourcing multiple choice science questions. In: Derczynski, L., Xu, W., Ritter, A., Baldwin, T. (eds.) Proceedings of the 3rd Workshop on Noisy User-generated Text, NUT@EMNLP 2017, Copenhagen, Denmark, September 7, 2017. pp. 94-106. Association for Computational Linguistics (2017). https://doi.org/10.18653/v1/w17-4413, https://doi.org/10.18653/ $\mathrm{v} 1 / \mathrm{w} 17-4413$

9. Westphal, O., Voigtländer, J.: Describing Console I/O Behavior for Testing Student Submissions in Haskell. In: Eighth and Ninth International Workshop on Trends in Functional Programming in Education, Proceedings. EPTCS, vol. 321, pp. 19-36. EPTCS (2020). https://doi.org/10.4204/EPTCS.321.2

10. Westphal, O., Voigtländer, J.: Implementing, and Keeping in Check, a DSL Used in E-Learning. In: Proceedings of the 15th International Symposium on Functional and Logic Programming. LNCS, vol. 12073. Springer (2020). https://doi.org/10.1007/978-3-030-59025-3_11 